\title{
Burning mouth syndrome: Correlation of treatment to clinical variables of the disease
}

\author{
Javier Silvestre-Rangil ${ }^{1}$, Francisco-Javier Silvestre ${ }^{2}$, Carmen Tamarit-Santafé ${ }^{3}$, Daniel Bautista ${ }^{4}$
}

\author{
${ }^{1}$ Collaborating dental surgeon, Stomatology Unit. Dr. Peset University Hospital (Valencia, Spain) \\ ${ }^{2}$ Assistant Professor of the Department of Stomatology, University of Valencia Medical and Dental School. Head of the Stomatol- \\ ogy Unit. Dr. Peset University Hospital (Valencia, Spain) \\ ${ }^{3}$ Associate Professor of Odontology in Special Patients. Cardenal Herrera-CEU University (Valencia, Spain) \\ ${ }^{4}$ Staff physician, Department of Preventive Medicine. Dr. Peset University Hospital (Valencia, Spain)
}

Correspondence:

Hospital Universitario Dr. Peset, Consultas Externas, C/ Juan de Garay s/n, 46017 Valencia (Spain)

Francisco.silvestre@uv.es

Received: 29/07/2010

Accepted: 08/12/2010

Silvestre-Rangil J, Silvestre FJ, Tamarit-Santafé C, Bautista D. Burning mouth syndrome: Correlation of treatment to clinical variables of the disease. Med Oral Patol Oral Cir Bucal. 2011 Nov 1;16 (7):e890-4. http://www.medicinaoral.com/medoralfree01/v16i7/medoralv16i7p890.pdf

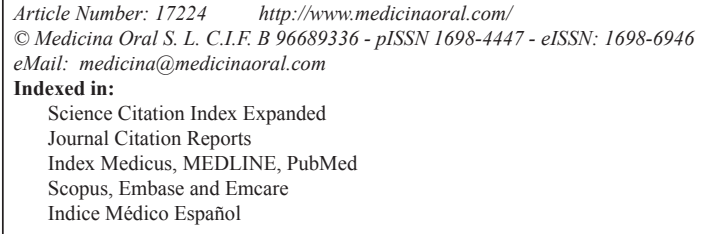

\begin{abstract}
Objective: Burning mouth syndrome (BMS) is a complex disorder with a still uncertain etiopathogenesis. A number of treatments have been used in application to BMS, though without clearly successful results. The present study compares the improvement in BMS obtained as a result of different treatment modalities in relation to the clinical characteristics of the patients.

Study Design: A retrospective cohort study was made of 115 patients with BMS (109 females and 6 males) subjected to different treatments with a view to improving the symptoms. The clinical variables examined included the duration of the disorder, the location of the burning sensation, its daily variations and relationship with meals. The parameters were measured using a visual analog scale (VAS) applied at baseline and again after two weeks of treatment.

Results: The mean patient age was $70 \pm 11.41$ years, and the mean duration of the syndrome was $7.16 \pm 2.63$ years. The tongue was the most frequently affected location. Anxiolytic treatment afforded the best results $(\mathrm{p}<0.001)$, and the patients with the shortest duration of disease showed the best improvement with treatment $(\mathrm{p}=0.005)$.

Conclusion: The greatest treatment efficacy corresponded to anxiolytic drugs, and treatment was more effective when introduced early after the diagnosis of BMS.
\end{abstract}

Key words: Burning mouth syndrome, glossodynia, stomatodynia, treatment, evolution. 


\section{Introduction}

Burning mouth syndrome (BMS) is characterized by continuous burning sensation located in the apparently normal oral mucosa. It can be accompanied by dry mouth (xerostomia), taste alterations and other anomalous sensations. The discomfort may be present from the morning or increase in the course of the day. The estimated prevalence of BMS is 0.7-4.6\%, and the condition mostly affects women of middle or advanced age (1). The etiopathogenesis of BMS remains unclear, and the different treatments used to date have shown little efficacy (2).

Psychological alterations such as anxiety and depression are often seen in these patients (3-7). Both processes can modulate pain perception, increasing or decreasing neuronal conduction from the peripheral receptors, and modifying individual perception of discomfort. On the other hand, patients with BMS have been found to improve of their symptoms with the use of anxiolytic drugs or psychotherapy (8).

On the other hand, patients with BMS have been found to experience alterations in heat tolerance, taste disturbances and increased palpebral reflex excitability. This suggests the presence of alterations at both central and peripheral nervous system level (9). Neuropathic mechanisms have been identified, showing symptoms typical of alterations in the trigeminal domain. The clinical characteristics of BMS in turn suggest the possible participation of neuronal inflammation in the etiopathogenesis of the syndrome, together with a series of other mechanisms $(9,10)$.

Many treatments have been recommended for improving the symptoms of BMS, including sialogogues, topical anesthetics in rinses, anxiolytic drugs, antidepressants, anticonvulsivants, alpha-lipoic acid and psychotherapy $(8,9-13)$, though no evidence has been produced by controlled clinical trials in support of such therapies. The best results have been reported for clonazepam, administered both topically and via the systemic route, though improvement has not been documented in all cases $(14,15)$.

The present study was designed to evaluate the improvement afforded by different treatments in one same cohort of patients with BMS seen in the University Dental Clinic (Valencia University Medical and Dental School, Valencia, Spain), and to explore the relationship between the treatment provided and the clinical characteristics of the syndrome, particularly as regards time.

\section{Material and Methods}

A retrospective cohort study was made of 115 patients with BMS (109 females and 6 males), seen in the University Dental Clinic for at least two years. Informed consent to participation in the study was obtained in all cases.
We included subjects with discomfort described as a burning or itching sensation of the oral mucosa for at least 6 months, and who presented no organic lesions at the time of initial exploration capable of accounting for the symptoms. Likewise, no prior laboratory test alterations (biochemistry and hematology) were observed. All the patients had been treated for their oral symptoms and had been subjected to follow-up. As regards treatment, patients with dry mouth received sialogogues and artificial saliva formulations, while another group received palliative rinses in the form of $2 \%$ lidocaine solution, another was prescribed anxiolytic drugs (in all cases ketazolam $30 \mathrm{mg}$ one capsule at bedtime), and another group received a topical $0.02 \%$ capsaicin formulation.

The following exclusion criteria were considered at the time of diagnosis: subjects who at exploration showed specific lesions or systemic disease capable of accounting for the oral burning sensation; patients in which the symptoms had been present for under 6 months; patients with a very poor general condition who could present some serious illness precluding adequate exploration and follow-up; and patients undergoing oncological treatment.

The personal and clinical data of each patient were recorded, with special emphasis on the duration of the syndrome, the location of the burning sensation in the oral cavity, the associated discomfort, daily variations and relationship with meals.

The intensity of discomfort was measured using a visual analog scale (VAS) scored from 0 to $10 \mathrm{~cm}$, where $0=$ no symptoms and $10=$ unbearable discomfort. The VAS was applied at baseline and again after two weeks of treatment.

\section{Results}

In this study, $92.17 \%$ of the patients with BMS were females, while only $7.82 \%$ were males (10/1 proportion). The mean age was $70.96 \pm 11.41$ years (range $37-98$ ).

(Table 1) shows the patient age distribution by decades. The overall mean duration of the disease was $7.16 \pm 2.63$ years (range 1-24)(percentile 50 corresponding to 5 years). Most of the patients $(90.4 \% ; n=104)$ referred no toxic habits. None of the subjects were regular alcohol consumers, and only 11 were smokers.

As regards medication history, 34 patients (29.6\%) were receiving no medication at the time of the first visit. In relation to the drugs used at the time of this visit $47.8 \%$ received anxiolytic drugs $(\mathrm{n}=55)$ and $21.7 \%$ antidepressants $(n=25)$. In turn, 29 patients used oral antidiabetic drugs and 26 were prescribed blood pressure-lowering medication.

The tongue was the most common location of burning sensation (56 patients), while the oral cavity in general was found to be affected in 24 subjects. Other locations 
Table 1. Patient age distribution by decades.

\begin{tabular}{|c|c|}
\hline Age & $\mathbf{n ( \% )}$ \\
\hline $30-39$ years & $2(1.73 \%)$ \\
\hline $40-49$ years & $4(3.4 \%)$ \\
\hline $50-59$ years & $15(13.04 \%)$ \\
\hline $60-69$ years & $24(20.86 \%)$ \\
\hline $70-79$ years & $43(37.39 \%)$ \\
\hline $80-89$ years & $19(16.52 \%)$ \\
\hline $90-99$ years & $8(6.95 \%)$ \\
\hline
\end{tabular}

were the lips, gums and cheek mucosa (20\%). Of note is the observation that the lower lip was affected in $10.4 \%$ of the cases.

The most frequent accompanying manifestation was dry mouth (xerostomia) $(64.3 \%)$, followed by taste alterations $(50.4 \%)$.

Regarding the treatment provided for BMS, 40 patients used anxiolytic drugs (34.8\%), 24 cases received 2\% lidocaine rinses (20.9\%), 22 used sialogogues (19.1\%), 11 used artificial saliva (9.6\%), and 18 patients used topical capsaicin gel (15.7\%).

The VAS score at baseline (before specific treatment) ranged from 6-10, while the most frequent score was 8 (mode). The scores were seen to decrease after treatment, with complete disappearance of discomfort in two cases. In the subjects administered anxiolytic treatment, the VAS scores decreased three or more points with respect to baseline - the difference versus the rest of treatments being statistically significant $(\mathrm{x} 2=32.29$, $\mathrm{p}<0.001$ ) (Fig. 1).

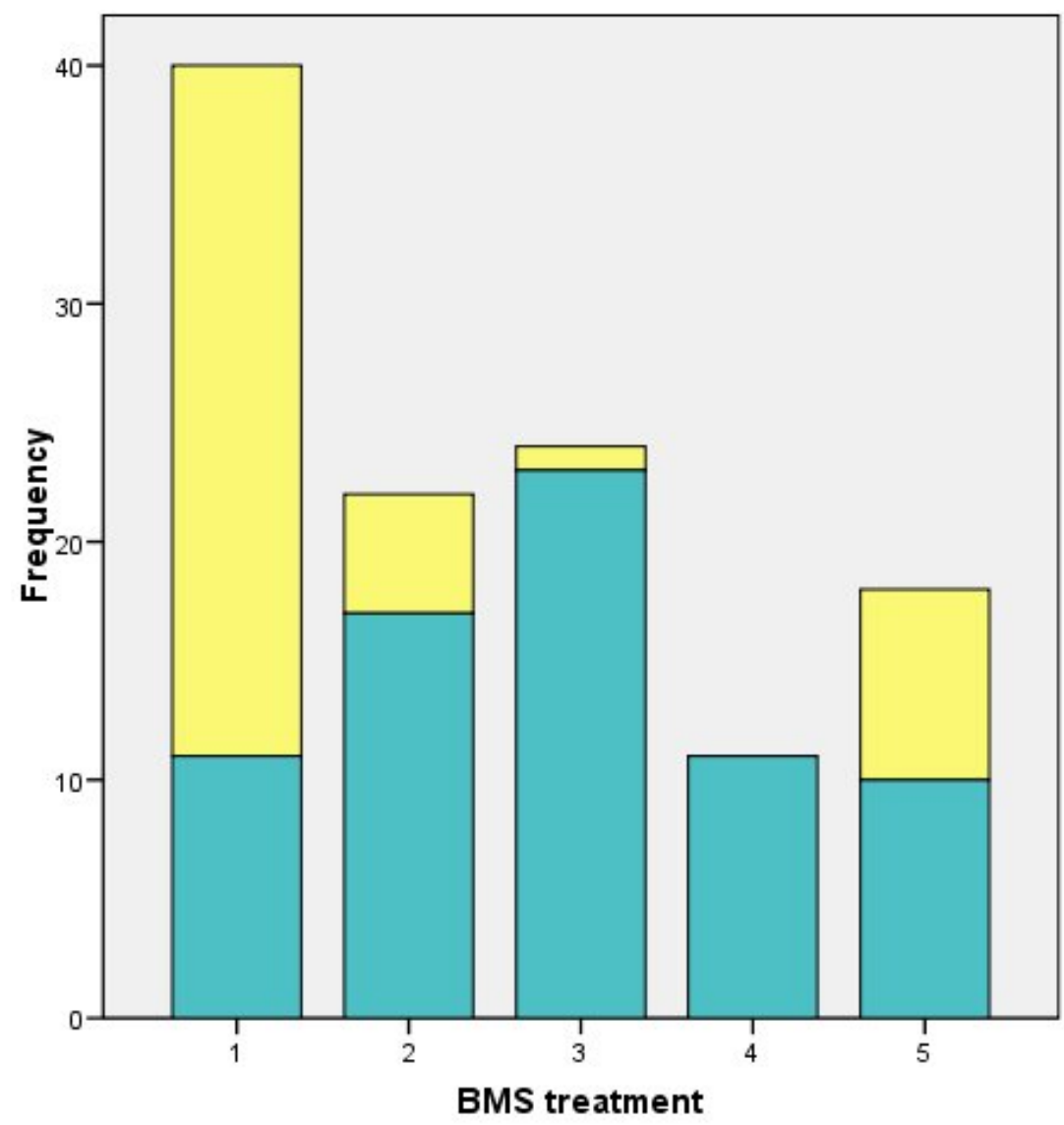

Score improvement

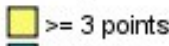

] 3 points

Fig. 1. Improvements in VAS symptoms score after treatment for burning mouth syndrome. Legends to columns:

1.Anxiolytics

1.Sialogogues

$3.2 \%$ lidocaine rinse

4.Artificial saliva

5.Topical capsaicin 
Score improvements of under three points were recorded in 72 cases $(62.6 \%)$, while improvements of three or more points were recorded in 43 patients $(37.4 \%)$ in the total study sample $(n=115)$.

No significant associations were observed between the variations in oral symptoms intensity and patient age or gender. Likewise, no significant relationship was noted between the variation in VAS score with specific treatment and other variables such as the location of burning sensation, the accompanying symptoms, daily variations or relationship with meals.

The patients with the shortest duration of BMS $(<2$ years) showed greater improvement with treatment $(>$ 4 points on the VAS)(Fisher's exact test, $\mathrm{p}=0.005$ ) (Fig. 2).

On examining the association between the variation in symptoms intensity and the type of medication administered, the greatest decrease in discomfort was seen to correspond to the patients administered anxiolytic drugs $(\mathrm{p}<0.01)$, followed by capsaicin $(\mathrm{x} 2=41.35$, $\mathrm{p}<0.01)$. No significant results were obtained in relation to the rest of treatments. Anxiolytics and capsaicin afforded a reduction in VAS score of three or more points in $72.5 \%$ and $44.4 \%$ of the patients administered these drugs, respectively.

\section{Discussion}

The results of this study show that the sooner treatment is prescribed after the diagnosis of burning mouth syndrome (BMS), the better the results obtained. In this sense, a mean improvement in VAS symptoms score of two points was recorded when the patients were treated in under four years after the diagnosis, and in one-third of the cases the improvement was three points or more. However, it must be taken into account that this was a retrospective study in which not all the treatments prescribed were equally effective in application to BMS. According to Sardella et al. (16), less than $30 \%$ of all patients experience improvement as a result of different treatments, and spontaneous remission in the first 5 years after the diagnosis is minimal. In our series, over $37 \%$ of the subjects experienced clear improvement ( $>3$ points on the VAS).

The management of BMS has been quite disappointing to date - this in part being due to our lack of knowledge of the specific mechanisms underlying the syndrome (14). Of the different treatments evaluated in our study, anxiolytic drugs were found to be the most effective option, affording a decrease in VAS score of three or more points in almost two-third of all cases, followed by topical capsaicin. Other authors $(1,15-17)$ have published controversial results with the administration of anxiolytic drugs.

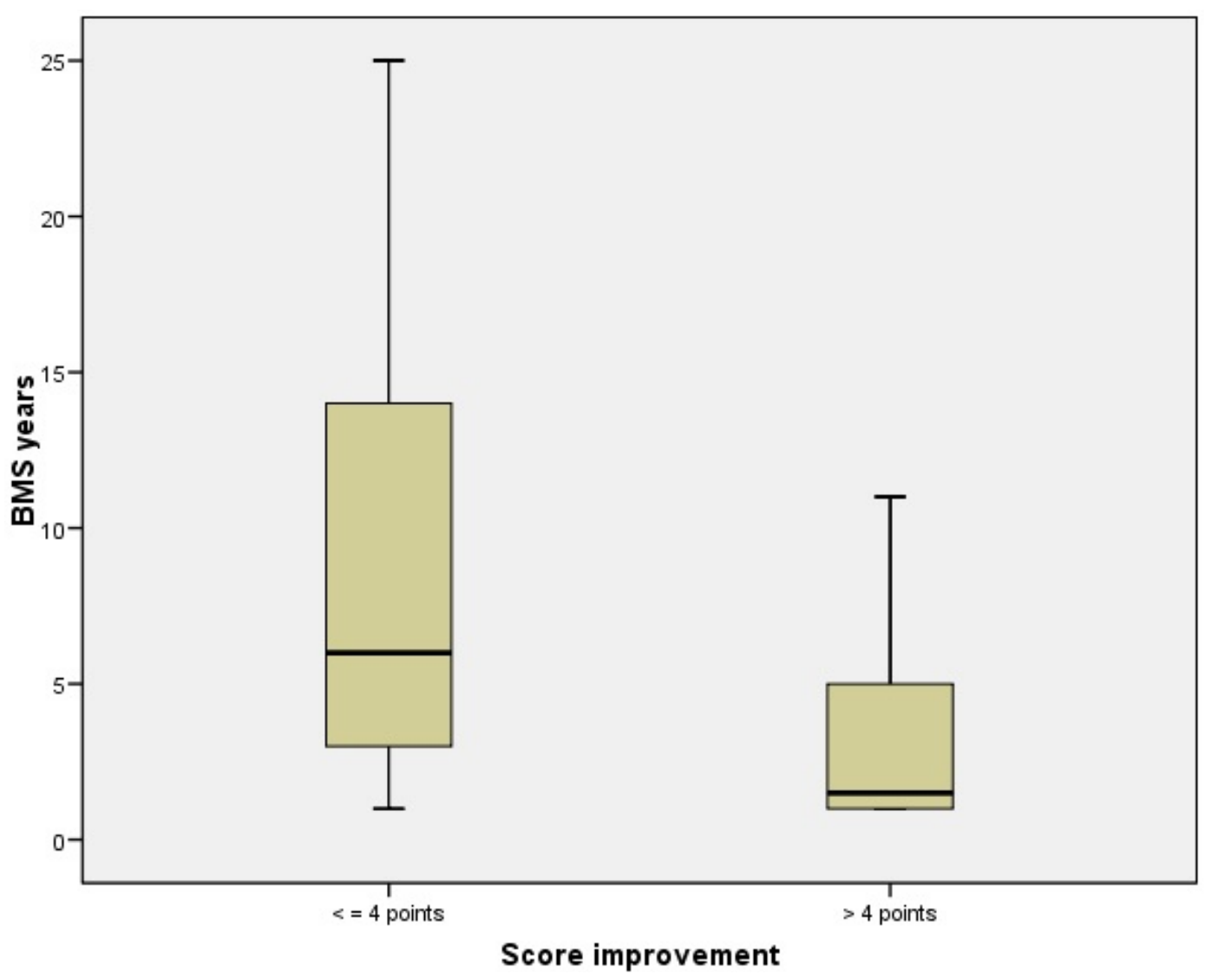

Fig. 2. Duration of burning mouth syndrome (years) in relation to symptoms improvement with treatment (over or under 4 points on the VAS). 
The highest incidence of BMS corresponded to elderly females with a mean age of almost 71 years. No direct relationship was found between improvement with therapy and patient age, though there was a tendency towards lesser improvement in very elderly women compared with younger females.

The most common systemic diseases in our series were psychiatric problems in the form of anxiety and depression, followed by arterial hypertension and type 2 diabetes. Although references have been made to BMS comorbidity, these conditions clearly can be explained by the advanced age of the patients.

The symptoms of BMS tend to become chronic. This complicates patient management and gives rise to situations similar to those found in chronic pain, where symptoms persistence over time gives rise to increased anxiety and depression.

Few studies have provided information on the spontaneous remission of BMS symptoms $(15,18,19)$, though in any case such remission must be very infrequent.

At present there is sufficient evidence of neuropathic involvement in this syndrome. Abnormal patient perception of certain nociceptive stimuli has been reported, with alterations of the trigeminal transmission small $\mathrm{C}$ fibers, and the appearance of inflammatory neuropeptides in the saliva of patients with BMS $(20,21)$. These findings suggest alterations in the sensory function of the trigeminal system in BMS. Likewise, an altered palpebral reflex has been reported, as well as extrapyramidal alterations with failure of dopaminergic inhibitory control in the basal ganglia (22).

On the other hand, patients with BMS are known to have an important presence of anxiety and depression, though it is difficult to establish Gao et al. (23), psychopathological factors together with local irritative elements may favor the appearance of BMS symptoms over time.

Treatment success has also been related to these etiopathogenic theories. Clonazepam has been found to be effective (both topically and via the systemic route) in improving the symptoms of BMS. Anxiolytic drugs are also able to improve the symptoms, as observed in our own patients.

Burning mouth is a complex clinical condition, though there is sufficient evidence to allow a more effective approach to the disease. In this sense, we consider that topical clonazepam should be combined with the use of anxiolytic drugs (ketazolam) in the presence of an important anxiolytic component, and that treatment should be started as soon as possible after the diagnosis of BMS.

\section{References with links to Crossref - DOI}

\section{References}

1. Scala A, Checchi L, Montevecchi M, Marini I, Giamberardino MA. Update on burning mouth syndrome: overview and patient management. Crit Rev Oral Biol Med. 2003;14:275-91.

2. Zakrzewska JM. The burning mouth syndrome remains an enigma. Pain. 1995;62:253-7.

3. Rojo L, Silvestre FJ, Bagan JV, De Vicente T. Prevalence of psychopathology in burning mouth syndrome. A comparative study among patients with and without psychiatric disorders and controls. Oral Surg Oral Med Oral Pathol. 1994;78:312-6.

4. Grushka M, Sessle BJ, Miller R. Pain and personality profiles in burning mouth syndrome. Pain. 1987;28:155-67.

5. Carlson CR, Miller CS, Reid KI. Psychosocial profiles of patients with burning mouth syndrome. J Orofac Pain. 2000;14:59-64.

6. Al Quran FA. Psychological profile in burning mouth syndrome. Oral Surg Oral Med Oral Pathol Oral Radiol Endod. 2004;97:33944.

7. Merigo E, Manfredi M, Zanetti MR, Miazza D, Pedrazzi G, Vescovi P. Burning mouth syndrome and personality profiles. Minerva Stomatol. 2007;56:159-67.

8. Jääskeläinen SK, Rinne JO, Forssell H, Tenovuo O, Kaasinen V, Sonninen P, et al. Role of the dopaminergic system in chronic pain - a fluorodopa-PET study. Pain. 2001;90:257-60.

9. Grémeau-Richard C, Dubray C, Aublet-Cuvelier B, Ughetto S, Woda A. Effect of lingual nerve block on burning mouth syndrome (stomatodynia): a randomized crossover trial. Pain. 2010;149:27-32. 10. Grushka M, Epstein J, Mott A. An open-label, dose escalation pilot study of the effect of clonazepam in burning mouth syndrome. Oral Surg Oral Med Oral Pathol Oral Radiol Endod. 1998;86:55761.

12. Maina G, Vitalucci A, Gandolfo S, Bogetto F. Comparative efficacy of SSRIs and amisulpride in burning mouth syndrome: a singleblind study. J Clin Psychiatry. 2002;63:38-43.

13. Femiano F, Gombos F, Scully C. Burning mouth syndrome: the efficacy of lipoic acid on subgroups. J Eur Acad Dermatol Venereol. 2004; 18:676-8

14. Miziara ID, Filho BC, Oliveira R, Rodrigues dos Santos RM. Group psychotherapy: an additional approach to burning mouth syndrome. J Psychosom Res. 2009;67:443-8.

15. Zakrzewska JM, Forssell H, Glenny AM. Interventions for the treatment of burning mouth syndrome. Cochrane Database Syst Rev. 2005;1:CD002779.

16. Sardella A, Lodi G, Demarosi F, Bez C, Cassano S, Carrassi A. Burning mouth syndrome: a retrospective study investigating spontaneous remission and response to treatments. Oral Dis. 2006;12:152-5.

17. Culhane NS, Hodle AD. Burning mouth syndrome after taking clonazepam. Ann Pharmacother. 2001;35:874-6.

18. Pinto A, Sollecito TP, DeRossi SS. Burning mouth syndrome. A retrospective analysis of clinical characteristics and treatment outcomes. N Y State Dent J. 2003;69:18-24.

19. Mínguez Serra MP, Salort Llorca C, Silvestre Donat FJ. Pharmacological treatment of burning mouth syndrome: A review and update. Med Oral Patol Oral Cir Bucal. 2007;12:E299-304.

20. Grushka M, Epstein JB, Gorsky M. Burning mouth syndrome. Am Fam Physician. 2002;65:615-20.

21. Zakrzewska JM. Facial pain: an update. Curr Opin Support Palliat Care. 2009;3:125-30.

22. Borelli V, Marchioli A, Di Taranto R, Romano M, Chiandussi S, Di Lenarda R, et al. Neuropeptides in saliva of subjects with burning mouth syndrome: a pilot study. Oral Dis. 2010;16:365-74.

23. Gao J, Chen L, Zhou J, Peng J. A case-control study on etiological factors involved in patients with burning mouth syndrome. J Oral Pathol Med. 2009;38:24-8. 